\title{
Has the time come for opportunistic oral cancer screening?
}

\author{
Opportunistic screening for oral cancer and precancer in general dental practice: results of a demonstration study \\ K. Lim, D. R. Moles, M. C. Downer and P. M. Speight Br Dent J 2003; 194: 497-502
}

\section{Objectives}

To demonstrate the feasibility of opportunistic oral cancer and precancer screening in general dental practice and to determine the prevalence of relevant lesions and risk habits in a population of general dental practice attenders.

\section{Design, setting and methods}

A prospective demonstration study, recruiting patients opportunistically in general dental practices. Eighteen general dental practitioners took part in this study. Each attended training sessions to be advised of the study protocol and the criteria of a positive and negative screen. Patients over the age of 35 years were prospectively and opportunistically recruited. Each patient was asked to complete a health questionnaire concerning age, gender, ethnicity, smoking and drinking habits. The dentist then examined the soft tissues and recorded the presence or absence of lesions independently on a second form. The forms were collated and data were analysed to determine prevalence of lesions and associations with risk habits.

\section{Results}

Data on 2,265 patients were available for analysis. Oral lesions were detected in 319 patients (14.1\%). Ninety-four patients (4.2\%) had lesions considered to be either malignant or potentially malignant. There was a significant association between positive lesions and male gender (IRR 1.86, 95\% CI 1.22-2.82), heavy smoking (males: IRR 3.68, 95\% CI 2.10-6.43: female; IRR 3.58, 95\% CI 1.35-9.50) and heavy alcohol use in males (IRR 2.98, 95\% CI 1.06-3.47).

\section{Conclusions}

The results suggest that patients attending general dental practices are representative of the general population both in terms of lesion prevalence and high risk habits such as smoking and drinking. This supports the view that opportunistic screening in a general dental practice setting may be a realistic alternative to population screening. Further research is needed to determine the cost effectiveness of this approach and to investigate the value of targeting high risk groups within this population. General dental practice is ideal for the evaluation of such systems prior to extending these studies to other healthcare settings.

\section{IN BRIEF}

- Oral cancer is an important disease that is easily identified in a dental practice setting.

- This study shows that GDPs can easily detect relevant lesions, using a simple systematic mucosal examination, with little disruption to a normal practice routine. The prevalence of relevant lesions and of risk habits among patients who attend general dental practices appears to be representative of the general population.

- The data suggest that opportunistic screening in a general dental practice setting, particularly if high risk groups can be targeted, might be a realistic option.

\section{COMMENT}

Of all oral diseases, oral cancers are the most life threatening. Further, the primary risk factors for these cancers - use of tobacco and alcohol products - have been known for decades. Yet dental schools have placed little emphasis on oral cancer prevention and early detection, especially compared with other content areas such as restorative and prosthetic dentistry. Not surprisingly, general dentists have essentially ignored detection of these cancers. Equally unsurprising, most oral cancers are detected at late stage and, as a result, five-year survival rates are among the lowest for all major cancers. Of all health providers, general dentists are the most logical group to screen for these cancers. But some argue that those persons at highest risk for oral cancers do not seek dental services. Moreover, the evidence to demonstrate the benefits of early cancer detection has been scant. As a result, few leading authorities suggest population screening for oral cancers. Others have suggested screening for oral cancers opportunistically especially when high-risk groups can be identified. But, here too, little evidence has been available to support the recommendation.

The study by Lim et al. is important because it demonstrates that opportunistic screening for oral cancers by general dentists is feasible and worthwhile. Here, opportunistic screening means examining patients who are at high risk for oral cancer because of risky behaviours, for soft tissue lesions. Moreover, this study demonstrates that the prevalence of positive lesions is consistent with the general population prevalence and that the dental attendees mimicked the general population in their use of tobacco and alcohol.

Eighteen general dentists were trained and standardized to identify erythroplakia (red lesions) and leukoplakia (white lesions), the primary lesions associated with being potentially malignant or malignant. This examination was in addition to their regular procedures and required about three minutes. Prior to the examination, patients completed a short survey regarding their age, sex, ethnicity and smoking and drinking practices.

This pivotal study determined, for the first time, that opportunistic screening in general dental practice is the place to screen for oral cancer because these investigators showed that the prevalence of lesions associated with oral cancers and the prevalence of risky behaviours (use of tobacco and/or alcohol) among dental attenders are similar to the general UK population. Thus, opportunistic screening by general dentists is a significant step forward in our efforts to decrease morbidity and mortality resulting from oral cancers.

\section{A. M. Horowitz, Senior Scientist,}

National Institute of Dental and Craniofacial Research, National Institutes of Health, Department of Health and Human Services Bethesda, MD USA 